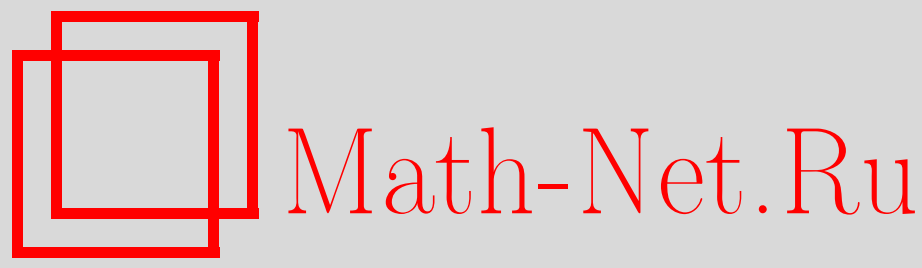

В. В. Чистяков, О квантовом аналоге неустойчивых предельных циклов периодически возмущаемого перевернутого осциллятора, ТМФ, 2019, том 198, номер 1, 19-31

DOI: https://doi.org/10.4213/tmf9541

Использование Общероссийского математического портала Math-Net.Ru подразумевает, что вы прочитали и согласны с пользовательским соглашением http://www . mathnet.ru/rus/agreement

Параметры загрузки:

IP: 54.198 .64 .247

26 апреля 2023 г., $14: 36: 35$

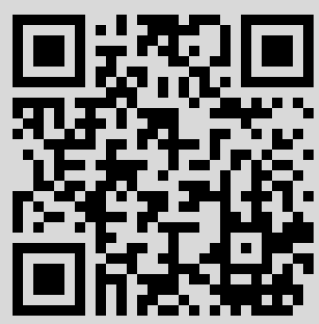




\title{
О КВАНТОВОМ АНАЛОГЕ НЕУСТОЙЧИВЫХ ПРЕДЕЛЬНЫХ ЦИКЛОВ ПЕРИОДИЧЕСКИ ВОЗМУЩАЕМОГО ПЕРЕВЕРНУТОГО ОСЦИЛЛЯТОРА
}

\begin{abstract}
С целью исследования квантового аналога для классических предельных циклов изучено поведение частицы в отрицательном квадратичном потенциале, возмущаемом синусоидальным полем. Предложен тип волновой функции, асимптотически удовлетворяющей оператору начальных условий и допускающей при этом аналитическое интегрирование временно́го уравнения Шредингера. Решение демонстрирует, что при определенных фазах возмущения, задающихся вынуждающей частотой и начальной неопределенностью координаты, центр волнового пакета временно стабилизируется вблизи максимума потенциала примерно на протяжении двух натуральных периодов осциллятора, после чего удаляется на бесконечность с бифуркацией направления ухода. Эффект не маскируется расплыванием пакета, так как на вышеозначенном временном интервале имеет место его (пакета) аномальное сужение (коллапс) до размера порядка характерной длины, лишь затем сменяющееся неограниченным расплыванием.
\end{abstract}

Ключевые слова: перевернутый квантовый осциллятор, периодическое возмущение, предельный цикл, нестационарное уравнение Шредингера, обобщенный гауссовский тип, коллапс, динамическая стабилизация, бифуркация.

DOI: https://doi.org/10.4213/tmf9541

\section{1. ВВЕДЕНИЕ}

Отрицательный квадратичный потенциал в одномерном варианте, или, иначе, перевернутый гармонический одномерный осциллятор, является элементарной моделью неустойчивых квантовых систем - распадающихся возбужденных молекул и комплексов, экситонных состояний в полупроводнике, различного рода систем

Работа выполнена при поддержке РФФИ (грант № 16-08-00997).

${ }^{*}$ Санкт-Петербургский национальный исследовательский университет информационных технологий, механики и оптики, Санкт-Петербург, Россия.

E-mail: v.chistyakov@corp.ifmo.ru 
с потенциальными барьерами и пр. [1]-[3]. Также модель дает интересные результаты в дискретном конфигурационном пространстве, когда разностное уравнение Шредингера допускает "удержание волн на потенциальном склоне” [4], [5]. $\mathrm{C}$ чисто фундаментальной точки зрения инвертированный гармонический осциллятор (ИГО) интересен в связи с определенными трудностями в плане реализации принципа соответствия между классической и квантовой теориями [6].

Разумеется, речь идет не о собственно модели ИГО, а предполагается временна́я зависимость гамильтониана, как динамическая, так и стохастического типа. Это может быть зависимость от времени $t$ собственно параметров модели - массы $m(t)$, "резонансной" частоты $\omega(t)$ [7], или же наличие температурного окружения [3], часто - присутствие в гамильтониане дополнительных слагаемых, отвечающих за диссипацию или переменную во времени нелинейность [8].

Особый интерес представляет наличие периодического внешнего воздействия в плане исследования поведения вышеупомянутых неустойчивых систем в поле лазерного излучения, или плазменных колебаний, а также при изучении туннелирования частиц сквозь квадратичный барьер в присутствии таких осциллирующих полей [9]. В последнем случае коэффициент прохождения существенно зависит от характеристик вынуждающего воздействия: его амплитуды, частоты и фазы.

В настоящей работе интегрируется и исследуется нестационарное уравнение Шредингера для квантовой частицы вблизи положения максимума отрицательного квадратичного потенциала в присутствии синусоидального во времени пространственнооднородного поля. Цель - установить возможность существования квантового аналога так называемой динамической стабилизации - неустойчивого предельного цикла классической частицы в поле перевернутого осциллятора при дополнительном его возмущении постоянной гармонической силы определенной фазы.

\section{2. ДИНАМИЧЕСКАЯ СТАБИЛИЗАЦИЯ В КЛАССИЧЕСКОЙ МЕХАНИКЕ}

Перевернутый гармонический осциллятор ( $\omega=1, m=1)$, периодически возмущаемый на безразмерной частоте $\Omega$, описывается функцией Гамильтона в безразмерных же координатах $H(p, q)=p^{2} / 2-q^{2} / 2-f_{0} \sin (\Omega \tau+\varphi) q$ и подчиняется уравнениям движения

$$
\frac{d}{d \tau}\left(\begin{array}{l}
q \\
p
\end{array}\right)=\left(\begin{array}{ll}
0 & 1 \\
1 & 0
\end{array}\right)\left(\begin{array}{l}
q \\
p
\end{array}\right)+f_{0} \sin (\Omega \tau+\varphi)\left(\begin{array}{l}
0 \\
1
\end{array}\right), \quad \tau \geqslant 0 .
$$

С учетом начальных условий по координате $q_{0}$ и импульсу $p_{0}$ решение имеет вид

$$
\begin{gathered}
q(\tau)=\frac{1}{2}\left(A_{0}+A_{f}\right) e^{\tau}+B_{f}(\tau)+\frac{1}{2}\left(C_{0}+C_{f}\right) e^{-\tau}, \\
A_{0}=p_{0}+q_{0}, \quad A_{f}=f_{0} \frac{\sin \varphi+\Omega \cos \varphi}{1+\Omega^{2}}, \\
p(\tau)=\frac{1}{2}\left(A_{0}+A_{f}\right) e^{\tau}+\dot{B}_{f}(\tau)-\frac{1}{2}\left(C_{0}+C_{f}\right) e^{-\tau}, \\
C_{0}=q_{0}-p_{0}, \quad C_{f}=f_{0} \frac{\sin \varphi-\Omega \cos \varphi}{1+\Omega^{2}}, \\
B_{f}(\tau)=-\frac{f_{0} \sin (\Omega \tau+\varphi)}{1+\Omega^{2}}, \quad \dot{B}_{f}(\tau)=-\frac{f_{0} \Omega \cos (\Omega \tau+\varphi)}{1+\Omega^{2}},
\end{gathered}
$$


и оно ограничено на положительной временно́й полуоси при выполнении соотношения

$$
A_{0}+A_{f}=0 \quad \Longleftrightarrow \quad p_{0}+q_{0}+f_{0} \frac{\sin \varphi+\Omega \cos \varphi}{1+\Omega^{2}}=0 .
$$

Преобразовывая, получаем условие так называемой динамической стабилизации перевернутого осциллятора внешней синусоидальной силой

$$
\begin{aligned}
& \sin \varphi+\Omega \cos \varphi=-\frac{\left(1+\Omega^{2}\right)\left(p_{0}+q_{0}\right)}{f_{0}} \Longrightarrow \\
& \Longrightarrow \varphi=-\arcsin \left(\frac{\sqrt{1+\Omega^{2}}\left(p_{0}+q_{0}\right)}{f_{0}}\right)-\operatorname{arctg} \Omega
\end{aligned}
$$

при дополнительном требовании на амплитуду последней

$$
f_{0} \geqslant \sqrt{1+\Omega^{2}}\left|p_{0}+q_{0}\right|
$$

Примечательно, что ограниченные решения (1) с различными начальными условиями, но равными значениями величины $p_{0}+q_{0}(2)$, имеют одинаковую асимптотику на больших временах, а потому аналогичную ей асимптотику должна иметь и любая их линейная комбинация.

В силу линейности динамических уравнений такая стабилизация имеет место, даже если возмущающий сигнал не гармонический, но сумма или интеграл таковых:

$$
F(q, t)=-q \int_{D} f_{0}(\Omega) \sin (\Omega \tau+\varphi(\Omega)) d \Omega, \quad D \subset \mathbb{R}^{+},
$$

со спектральными амплитудами $f_{0}(\Omega)$ и фазами $\varphi(\Omega)$, удовлетворяющими условиям (3) и выражению

$$
p_{0}+q_{0}+\int_{D} \frac{\sin \varphi(\Omega)+\Omega \cos \varphi(\Omega)}{1+\Omega^{2}} f_{0}(\Omega) d \Omega=0 .
$$

В частности, для "половины” состояний с нулевой начальной энергией и начальным условием (НУ) вида $p_{0}+q_{0}=0$ нет ограничения $(3)$ на вид $f_{0}(\Omega)$, а условие стабилизации упрощается:

$$
\int_{D} \frac{\sin \varphi(\Omega)+\Omega \cos \varphi(\Omega)}{1+\Omega^{2}} f_{0}(\Omega) d \Omega=0 .
$$

Если, например, выбирать фазовые сдвиги по закону $\varphi(\Omega)=-\operatorname{arctg} \Omega$, что обеспечивает автоматическое выполнение условия (3) при любых амплитудах $f_{0}(\Omega)$, то в этом случае

$$
\begin{aligned}
F(q, t) & =-q \int_{D} f_{0}(\Omega) \sin (\Omega \tau+\varphi(\Omega)) d \Omega= \\
& =-q \int_{D} f_{0}(\Omega)(\sin \Omega \tau \cos \varphi(\Omega)+\cos \Omega \tau \sin \varphi(\Omega)) d \Omega= \\
& =-q \int_{D} f_{0}(\Omega) \cos \varphi(\Omega)(\sin \Omega \tau-\Omega \cos \Omega \tau) d \Omega \Longrightarrow \\
\Longrightarrow \quad F(q, t) & =-q \int_{D} \frac{f_{0}(\Omega)}{\sqrt{1+\Omega^{2}}}(\sin \Omega \tau-\Omega \cos \Omega \tau) d \Omega,
\end{aligned}
$$




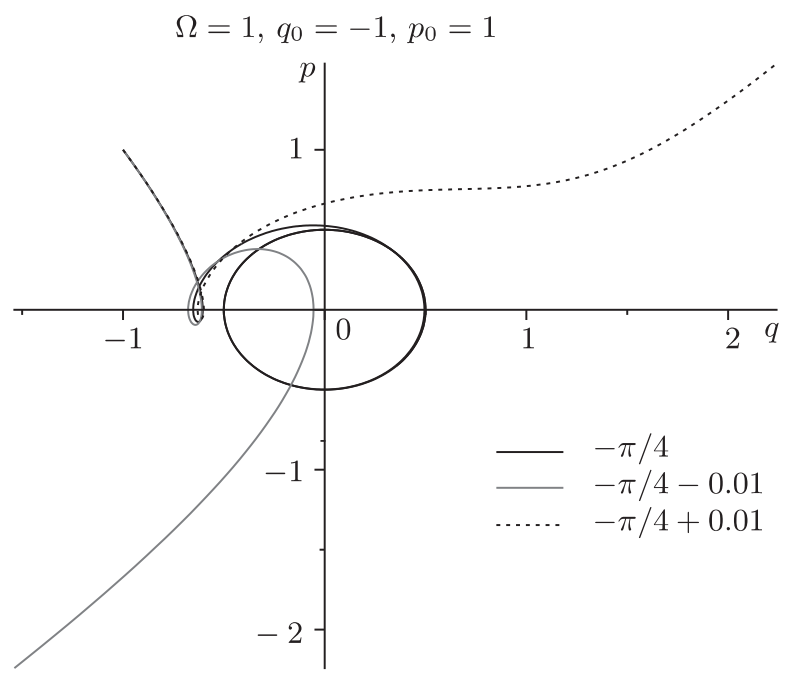

Рис. 1. Фазовые траектории классического инвертированного осциллятора для $\Omega=1$ при стабилизирующей фазе воздействия $\varphi=-\pi / 4$ и фазах, близких к ней, стартующие из одной точки.

где $f_{0}(\Omega)$ - произвольная обеспечивающая равномерную сходимость интеграла в $(6)$ на временно́й полуоси амплитуда.

В простейшем случае синусоидального воздействия на "резонансной" частоте $\Omega=1$ и с $f_{0}=1$ для стабилизирующей фазы $\varphi=-\pi / 4$ траектория в фазовом пространстве приближалась к предельному циклу, в то время как для близких фаз $-\pi / 4 \pm 0.01$ при тех же НУ $q_{0}=-1, p_{0}=1$ получались уходящие в противоположные бесконечности кривые (см. рис. 1$)$.

Таким образом, динамическая стабилизация перевернутого гармонического осциллятора в классической механике теоретически возможна для широкого класса вынуждающих усилий как при нулевой начальной энергии $E\left(p_{0}, q_{0}\right)=0$ и $p_{0}+q_{0}=0$, так и при $E\left(p_{0}, q_{0}\right) \neq 0\left(p_{0}+q_{0} \neq 0\right)$, но при выполнении условия $(3)$.

\section{3. УРАВНЕНИЕ ШРЕДИНГЕРА}

В безразмерных координатах $\xi=x \sqrt{m \omega / \hbar}, f_{0}=F_{0} /(\omega \sqrt{\hbar m \omega}), \tau=\omega t$ нестационарное уравнение Шредингера для периодически возмущаемого ИГО имеет вид

$$
i \frac{\partial \Psi(\xi, \tau)}{\partial \tau}=-\frac{1}{2} \frac{\partial^{2} \Psi(\xi, \tau)}{\partial \xi^{2}}-\frac{\xi^{2}}{2} \Psi(\xi, \tau)-f_{0} \xi \sin (\Omega \tau+\phi) \Psi(\xi, \tau) .
$$

Сдвиговая замена $\rho=\xi+f_{0} \sin (\Omega \tau+\phi)$ приводит к исчезновению линейного члена в потенциальной энергии, а представление волновой функции (в. ф.) $\Psi(\rho, \tau)=$ $\psi_{0}(\rho) g(\rho, \tau)$ в новом наборе переменных $(\rho, \tau)$, где (в свою очередь)

$$
g(\rho, \tau)=\exp \left(-i\left(E(\rho)+\frac{f_{0}^{2}}{4}\right) \tau+i \frac{f_{0}^{2} \sin (2 \tau+2 \phi)}{8}\right) z(\rho, \tau),
$$


а $E(\rho)=-1 /\left(2 \psi_{0}(\rho)\right) d^{2} \psi_{0}(\rho) / d \rho^{2}-\rho^{2} / 2$ (см. ниже), преобразует его к виду

$$
\begin{aligned}
& i \frac{\partial z(\rho, \tau)}{\partial \tau} \frac{1}{z}+\left(\frac{i}{z} \frac{\partial z(\rho, t)}{\partial \rho}+\frac{d E}{d \rho} \tau\right) f_{0} \Omega \cos (\Omega \tau+\phi)+ \\
& \quad+i \frac{d \psi_{0}(\rho)}{d \rho} \frac{1}{\psi_{0}} f_{0} \Omega \cos (\Omega \tau+\phi)+\frac{1}{z} \frac{\partial z(\rho, \tau)}{\partial \rho}\left(-i \frac{d E(\rho)}{d \rho} \tau\right)+ \\
& \quad+\frac{1}{2 z} \frac{\partial^{2} z(\rho, \tau)}{\partial \rho^{2}}-\frac{1}{2}\left(\frac{d E(\rho)}{d \rho}\right)^{2} \tau^{2}-\frac{i}{2} \frac{d^{2} E(\rho)}{d \rho^{2}} \tau+ \\
& \quad+\frac{1}{z} \frac{\partial z(\rho, t)}{\partial \rho} \frac{d \psi}{d \rho} \frac{1}{\psi}+\left(-i \frac{d E(\rho)}{d \rho} \tau\right) \frac{d \psi}{d \rho} \frac{1}{\psi}=0 .
\end{aligned}
$$

В уравнении $(7) \psi_{0}(\rho)=\psi_{0}\left(\xi+f_{0} \sin \phi\right)$ - в. ф. исходного состояния ИГО, которая должна в идеале быть собственной функцией для оператора НУ $\widehat{I}_{\mathrm{c}}=\widehat{p}+\widehat{\xi}=$ $-i \partial / \partial \xi+\xi$. Однако такая в. ф. $\psi_{\text {eig }}=C e^{-i \xi^{2} / 2}$, не будучи нормированной, не отвечает никакому физическому состоянию. Поэтому удовлетворить НУ можно лишь приближенно, выбрав, например, функцию обобщенного гауссовского типа с аномально большой полушириной пакета $\sigma_{0}=\sqrt{\overline{\xi^{2}(0)}} \gg 1$ :

$$
\begin{gathered}
\Psi(\xi, 0)=\psi_{0}(\xi)=\left(\frac{1}{\sqrt{2 \pi} \sigma_{0}}\right)^{1 / 2} \exp \left(-\frac{i \xi^{2}}{2}-\frac{\xi^{2}}{4 \sigma_{0}^{2}}\right), \\
\xi=\left.\left(\rho-f_{0} \sin (\omega \tau+\phi)\right)\right|_{\tau=0}=\rho-f_{0} \sin \phi
\end{gathered}
$$

которая описывает сходящийся к центру $\xi=0$ симметричный поток плотности вероятности, т. е. своего рода одномерный сходящийся к центру сферический пучок.

Такой выбор исходного волнового пакета обладает критическим преимуществом по сравнению с любыми другими: уравнение Шредингера становится интегрируемым аналитически. В самом деле, для $\psi_{0}(\xi)(8)$ следующие четыре величины:

$$
\begin{gathered}
\frac{d \psi_{0}(\rho)}{d \rho} \frac{1}{\psi_{0}(\rho)}=-\left(\rho-f_{0} \sin \phi\right)\left(\frac{1}{2 \sigma_{0}^{2}}+i\right) \\
E(\rho)=-\frac{1}{2}\left(\frac{1}{2 \sigma_{0}^{2}}+i\right)^{2}\left(\rho-f_{0} \sin \phi\right)^{2}-\frac{1}{2}\left(-\frac{1}{2 \sigma_{0}^{2}}-i\right)-\frac{\rho^{2}}{2}= \\
=-\frac{\rho^{2}}{2}\left(\frac{1}{4 \sigma_{0}^{4}}+\frac{i}{\sigma_{0}^{2}}\right)+\left(\frac{1}{4 \sigma^{4}}-1+\frac{i}{\sigma_{0}^{2}}\right) \rho f_{0} \sin \phi- \\
-f_{0}^{2} \sin ^{2} \phi\left(\frac{1}{8 \sigma_{0}^{4}}-\frac{1}{2}\right)+\frac{1}{4 \sigma_{0}^{2}}+i\left(\frac{1}{2}-\frac{f_{0}^{2} \sin ^{2} \phi}{2 \sigma_{0}^{2}}\right), \\
\frac{d E}{d \rho}=-\rho\left(\frac{1}{4 \sigma_{0}^{4}}+\frac{i}{\sigma_{0}^{2}}\right)+\left(\frac{1}{4 \sigma_{0}^{4}}-1+\frac{i}{\sigma_{0}^{2}}\right) f_{0} \sin \phi, \quad \frac{d^{2} E}{d \rho^{2}}=-\left(\frac{i}{\sigma_{0}^{2}}+\frac{1}{4 \sigma_{0}^{4}}\right)
\end{gathered}
$$

и зависящие от них коэффициенты в (8) суть многочлены степени не выше второй по $\rho$, и уравнение (9) можно решить через подстановку

$$
z(\rho, \tau)=e^{\alpha(\tau)+\beta(\tau) \rho+\gamma(\tau) \rho^{2}}, \quad \alpha(0)=\beta(0)=\gamma(0)=0,
$$

- своеобразный функциональный метод неопределенных коэффициентов. 
Это означает фундаментальное сохранение типа волны: будучи при $\tau=0$ зависимостью обобщенного гауссовского типа, эволюционирующая в. ф. квантового перевернутого осциллятора неизменно остается такой и при $\tau>0$. Тогда решение нестационарного уравнения Шредингера (УШ) сводится к интегрированию системы обыкновенных дифференциальных уравнений первого порядка.

Таким образом, квантовый линейный перевернутый осциллятор являет собой интегрируемую систему при любых допустимых временных зависимостях его параметров (и даже при наличии дополнительных членов в гамильтониане первого или второго порядков по координате и импульсу), но если только в качестве НУ был выбран волновой пакет обобщенного гауссовского типа. А в силу линейности нестационарного УШ в качестве стартового пакета можно также выбирать любые линейные комбинации таких функций, составляющих к тому же один класс с гауссовскими вейвлетами, используемыми для разложения/преобразования самых различных функций при обработке изображений и пр. Последнее означает, что к услугам теоретиков, эксплуатирующих модель перевернутого осциллятора, предоставлен весь накопленный за десятилетия богатый арсенал вычислительных алгоритмов, процедур и программ.

\section{4. ЭВОЛЮЦИЯ ПОЛУШИРИНЫ ВОЛНОВОГО ПАКЕТА}

Приравнивание в правых и левых частях (12) коэффициентов при одинаковых степенях $\rho$ приводит к системе трех обыкновенных дифференциальных уравнений

$$
\begin{aligned}
\dot{\gamma}(\tau)= & 2 \gamma(\tau)\left[-\left(\frac{i}{\sigma_{0}^{2}}+\frac{1}{4 \sigma_{0}^{4}}\right) \tau-i\left(\frac{1}{2 \sigma_{0}^{2}}+i\right)\right]+2 i \gamma^{2}(\tau)- \\
& -\frac{i}{2}\left(\frac{1}{4 \sigma_{0}^{4}}+\frac{i}{\sigma_{0}^{2}}\right)^{2} \tau^{2}+\left(\frac{1}{4 \sigma_{0}^{4}}+\frac{i}{\sigma_{0}^{2}}\right)\left(\frac{1}{2 \sigma_{0}^{2}}+i\right) \tau, \\
\dot{\beta}(\tau)= & i \beta(\tau)(2 i \tau-1+2 \gamma(\tau))+(2 \tau-i-2 \gamma(\tau)) f_{0} \Omega \cos (\Omega \tau+\phi)+f_{0} \sin \phi \tau, \\
\dot{\alpha}(\tau)= & i \gamma(\tau)+\frac{i}{2}\left(\frac{i}{4 \sigma_{0}^{4}}-\frac{1}{\sigma_{0}^{2}}\right) \tau-f_{0} \Omega \beta(\tau)(\cos (\Omega \tau+\varphi)+\tau \sin \varphi)- \\
& -i f_{o}^{2} \sin \varphi \Omega \tau \cos (\Omega \tau+\varphi)-\frac{i}{2} \tau^{2} f_{o}^{2} \sin ^{2} \varphi .
\end{aligned}
$$

Система последовательно интегрируется, и, в частности, для старшего коэффициента-функции получается выражение

$$
\begin{aligned}
\gamma(\tau) & =\frac{1}{4 \sigma_{0}^{2}}+\frac{i}{2}+\left(\frac{1}{2 \sigma_{0}^{2}}-\frac{i}{8 \sigma_{0}^{4}}\right) \tau+\frac{1}{2} \operatorname{tg}\left(2 i \tau-\operatorname{arctg}\left(i+\frac{1}{2 \sigma_{0}^{2}}\right)\right) \Longleftrightarrow \\
& \Longleftrightarrow\left\{\begin{array}{l}
\operatorname{Re} \gamma(\tau)=\frac{1}{4 \sigma_{0}^{2}}+\frac{\tau}{2 \sigma_{0}^{2}}-\frac{\sigma_{0}^{2}}{\operatorname{sh}^{2} 2 \tau+4 \sigma_{0}^{4} e^{-4 \tau}} \\
\operatorname{Im} \gamma(\tau)=-\frac{\tau}{8 \sigma_{0}^{4}}+\frac{\operatorname{sh}^{2} 2 \tau}{4 \sigma_{0}^{4} e^{-4 \tau}+\operatorname{sh}^{2} 2 \tau} .
\end{array}\right.
\end{aligned}
$$

С учетом квадратичного по $\rho$ члена в комплексной “энергии” $E(\rho)$, присутствующей под экспонентой в (6), а также аналогичного члена в начальной в. ф. (8) получается следующий действительный множитель при $\rho^{2}$ :

$$
-\frac{1}{4 \sigma(\tau)^{2}}=\frac{1}{4 \sigma_{\alpha}^{2}}+\frac{\tau / \alpha}{2 \sigma_{0}^{2}}-\frac{\tau / \sigma_{0}^{2}}{2 \sigma_{0}^{2}}-\frac{\sigma_{0}^{2}}{\operatorname{sh}^{2} 2 \tau+4 \sigma_{0}^{4} e^{-4 \tau}}-\frac{1}{4 \sigma_{0}^{2}},
$$




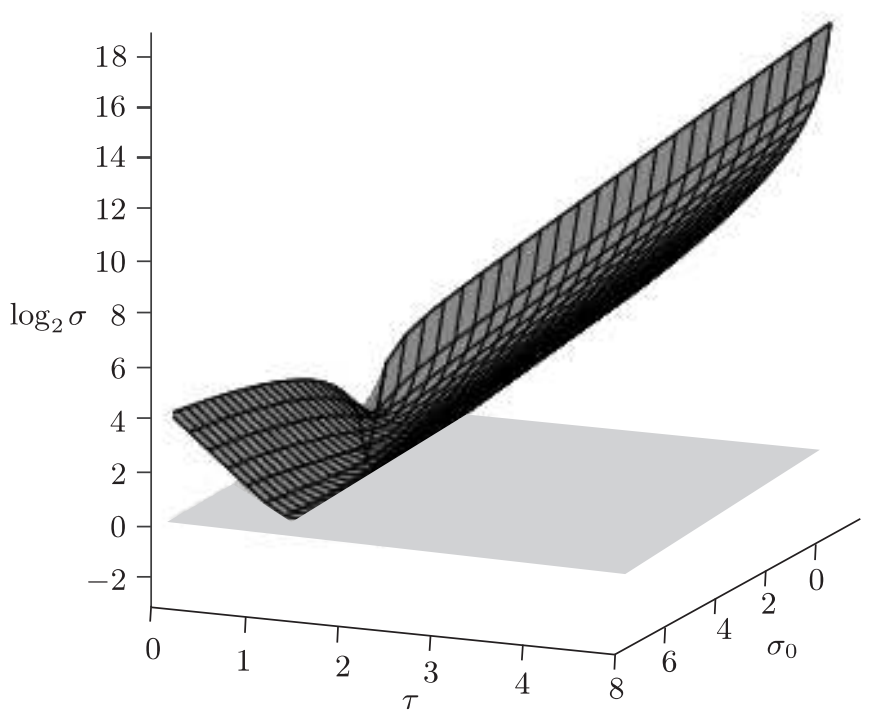

Рис. 2. Эволюция логарифма полуширины волнового пакета $\log _{2} \sigma\left(\tau, \sigma_{0}\right)$ при малых $\sigma_{0}$ (горизонтальная плоскость задана равенством $\sigma=1 / 2$ ).

определяющий временну́ю зависимость для полуширины пакета

$$
\sigma\left(\tau, \sigma_{0}\right)=\sigma_{0} \sqrt{e^{-4 \tau}\left(1+\frac{1}{16 \sigma_{0}^{4}}\right)-\frac{1}{8 \sigma_{0}^{4}}+\frac{e^{4 \tau}}{16 \sigma_{0}^{4}}} .
$$

Формула описывает стартовое убывание разброса $\sigma(\tau)$ на временах

$$
\tau \leqslant \tau_{0}=0.125 \ln \left(16 \sigma_{0}^{4}+1\right)
$$

до слабо зависящего от $\sigma_{0}$ минимального значения

$$
\sigma_{\min }=\sqrt{\sqrt{\frac{1}{4}+\frac{1}{64 \sigma_{0}^{4}}}-\frac{1}{4}} \approx \frac{1}{2}+\frac{1}{64 \sigma_{0}^{4}}, \quad \sigma_{0} \gg 1,
$$

а затем - его (разброса) восстановление при $\tau_{2}=2 \tau_{0}$ с последующим неограниченным и необратимым возрастанием по экспоненциальному закону (см. рис. 2).

Условие хотя бы какого-то сужения (компактизации) исходного волнового пакета (8) имеет вид $\sigma_{\min }<\sigma_{0}$,

$$
\begin{aligned}
-\frac{1}{4}+\sqrt{\frac{1}{4}+\frac{1}{64 \sigma_{0}^{4}}}<\sigma_{0}^{2} & \Longleftrightarrow \frac{1}{4}+\frac{1}{64 \sigma_{0}^{4}}<\sigma_{0}^{4}+\frac{\sigma_{0}^{2}}{2}+\frac{1}{16} \Longleftrightarrow \\
& \Longleftrightarrow 64 \sigma_{0}^{8}+32 \sigma_{0}^{6}-\frac{3 \sigma_{0}^{4}}{16}-\frac{1}{64}>0,
\end{aligned}
$$

и дает приближенно оценку $\sigma_{0}>0.51427360$. 
Что касается мнимой части временно́го множителя при $\rho^{2}$, определяющего внутренний поток перераспределения плотности вероятности в пакете, то с учетом тех же вкладов от начальной в. ф. и комплексной “энергии” $E$ (10) получается выражение

$$
\mu(\tau)=-\frac{\tau}{8 \sigma_{0}^{4}}+\frac{\operatorname{sh}^{2} 2 \tau}{4 \sigma_{0}^{4} e^{-4 \tau}+\operatorname{sh}^{2} 2 \tau}-\frac{1}{2}+\frac{\tau}{8 \sigma_{0}^{4}}=\frac{\operatorname{sh}^{2} 2 \tau}{4 \sigma_{0}^{4} e^{-4 \tau}+\operatorname{sh}^{2} 2 \tau}-\frac{1}{2} .
$$

Стартуя от уровня $-1 / 2$, множитель $\mu(\tau)$ ведет себя пороговым образом, обращаясь в нуль при $\tau_{1}=0.25 \log _{2}\left(1+4 \sigma_{0}^{2}\right)$, практически сливающимся с $\tau_{0}$ для $\sigma_{0} \gg 1$, и затем быстро стремясь к $+1 / 2$. Это означает, что внутренний поток вероятностной плотности инвертируется и становится расходящимся от центра: происходит внутреннее отражение волны и одновременно начинается ее необратимая деградация в сторону полной размытости в одномерном конфигурационном пространстве.

Таким образом, для как можно более точного соблюдения квантового аналога начальных условий необходимо выбрать волновой пакет обобщенного (в смысле комплексности параметров) гауссовского вида и как можно большой, почти макроскопической ширины. Независимо от любого линейного по координате воздействия такой пакет уже на старте коллапсирует в малый интервал размером около элементарной длины $\sqrt{\hbar / m \omega}$ за время порядка $\sim \log _{2}\left(\sigma_{0} / \omega\right)$, а затем необратимо расплывается по экспоненциальному закону. Потому о динамической стабилизации центра пакета $\overline{\widehat{x}(\tau)}$ имеет смысл говорить именно при выбранных (либо им равноценным) НУ и лишь в вышеозначенном временном интервале, за границей которого его (центра) положение не представляет интереса ввиду быстрого расплывания волны вдоль всей оси. Однако такой интервал может оказаться вполне достаточным для проявления эффекта на высокой частоте периодического воздействия $\Omega \gg \omega$, например, поля лазерного излучения, действующего на заряженную частицу в нестабильном состоянии с реальным временем распада.

\section{5. ДВИЖЕНИЕ ЦЕНТРА МАСС ВОЛНОВОГО ПАКЕТА}

Решение второго уравнения в (13) дает для коэффициента при первой степени $\rho$ формулу

$$
\begin{aligned}
& \beta(\tau)=-f_{0}\left(\cos \left(2 i \tau-\operatorname{arctg}\left(i+\frac{1}{2 \sigma^{2}}\right)\right)\right)^{-i / 2} \times \\
& \times \int_{0}^{\tau} \frac{\operatorname{tg}\left(2 i \tau^{\prime}-\operatorname{arctg}\left(i+\frac{1}{2 \sigma^{2}}\right)\right)}{\left(\cos \left(2 i \tau^{\prime}-\operatorname{arctg}\left(i+\frac{1}{2 \sigma^{2}}\right)\right)\right)^{-i / 2}}\left(\Omega \cos \left(\Omega \tau^{\prime}+\varphi\right)+\sin (\varphi) \tau^{\prime}\right) d \tau^{\prime} .
\end{aligned}
$$

Выделение в (18) действительной и мнимой частей приводит к чрезвычайно длинным и громоздким для представления выражениям, к тому же все равно не допускающим интегрирование в элементарных функциях и последующий их анализ. Автор решал численно эквивалентную (13) систему из шести уравнений относительно действительных и мнимых частей функций-коэффициентов $\alpha(\tau), \beta(\tau)$ и $\gamma(\tau)$.

Положение центра волнового пакета $\overline{\xi(\tau)}=\overline{\widehat{x}(\tau)} \sqrt{m \omega / \hbar}$ определяется стандартно через выделение полного квадрата в выражении

$$
-\frac{\rho^{2}}{4 \sigma(\tau)^{2}}+\operatorname{Re} \beta(\tau) \rho=-\frac{\left(\rho-2 \sigma(\tau)^{2} \operatorname{Re} \beta(\tau)\right)^{2}}{4 \sigma(\tau)^{2}}+(\sigma(\tau) \operatorname{Re} \beta(\tau))^{2}
$$




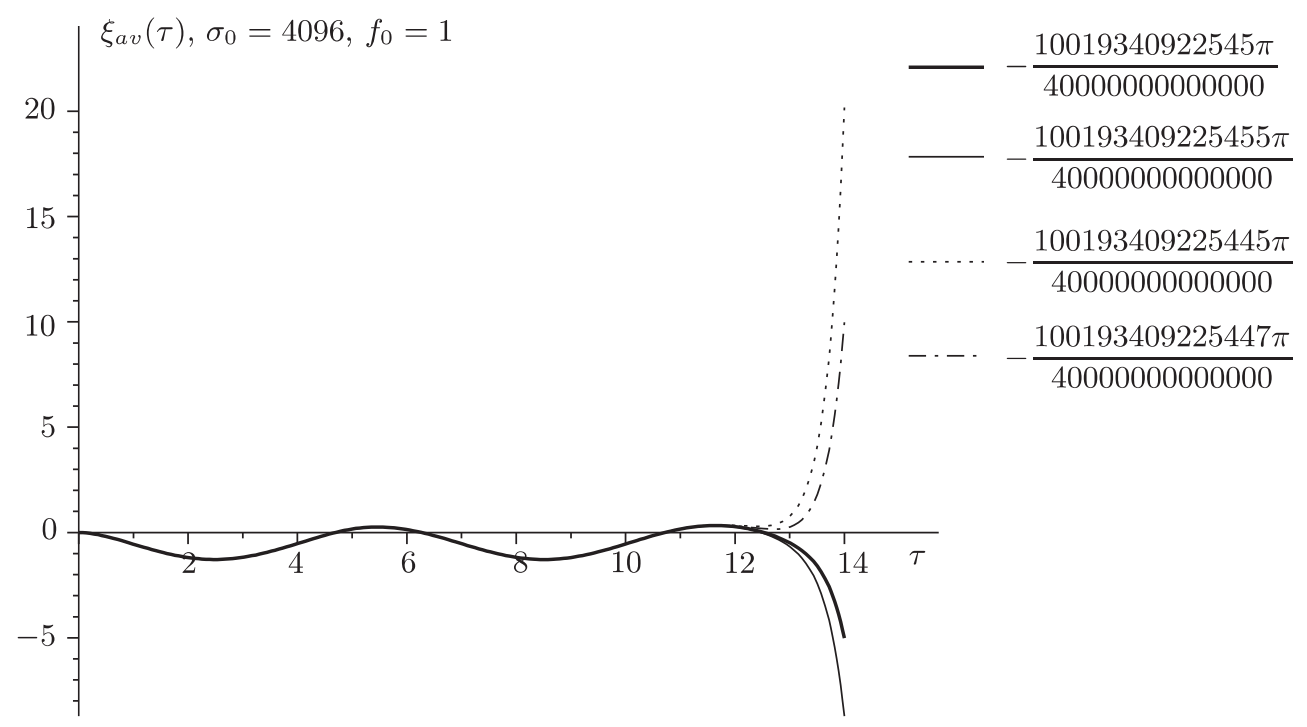

Рис. 3. Вре́менная стабилизация центра пакета $\overline{\xi(\tau)}$ с начальной полушириной $\sigma_{0}=2^{12}$ и бифуркация направления ухода при "натуральной" частоте $(\Omega=1$, дроби суть фазы $\varphi)$.

и дает равенство

$$
\overline{\rho(\tau)}=2 \sigma(\tau)^{2} \operatorname{Re} \beta(\tau) \quad \Longrightarrow \quad \overline{\xi(\tau)}=2 \sigma(\tau)^{2} \operatorname{Re} \beta(\tau)-f_{0} \sin (\Omega \tau+\varphi) .
$$

В общем случае произвольной начальной фазы $\varphi$ периодического воздействия центр пакета удалялся значительно от центра уже в течение первого основного "периода" осциллятора и на момент максимальной компактности находился от нулевого положения на расстоянии, на много порядков превышающем $\sigma_{\min }(16)$.

Однако путем вычислительного эксперимента для различных начальных разбросов $\sigma_{0}=2^{-3} \div 2^{21}$ найдены фазы в широком интервале частот $\Omega=2^{-7} \div 2^{7}$, при которых имели место (см. рис. 3-6)

а) негармонические колебания центра пакета около некоторой линии в течение примерно двух основных "периодов" времени, т. е. $0 \leqslant \tau<11 \div 14 \approx 2 \cdot 2 \pi$, затем резкий уход в сторону бесконечности;

б) бифуркации направления ухода центра пакета $\overline{\xi(\tau)}$ в положительную либо отрицательную полуось при изменении фазы $\varphi$ на пренебрежимо малую величину порядка $10^{-15 \div-20}$;

в) особенность поведения $\overline{\xi(\tau)}$ в окрестности момента разворота потока $\tau_{1}$, т. е. вблизи точки коллапса (см. рис. 4,5 , кривые $\ln \sigma(\tau)(15)$ и $\mu(\tau)(17))$.

Более детальный анализ окрестности точки $\tau_{1}$ инверсии для внутреннего потока вероятности для различных вынуждающих частот $\Omega=2^{3} \div 2^{7}$ показывает почти скачкообразное изменение фазы колебаний $\overline{\xi(\tau)}$ примерно на полупериод. Центр масс волнового пакета, начав двигаться в ожидаемом направлении, как бы "передумывает" и движется в противоположном. Положение уровня стабилизации, вокруг которого колеблется центр пакета, при этом дополнительно удаляется от нулевого 


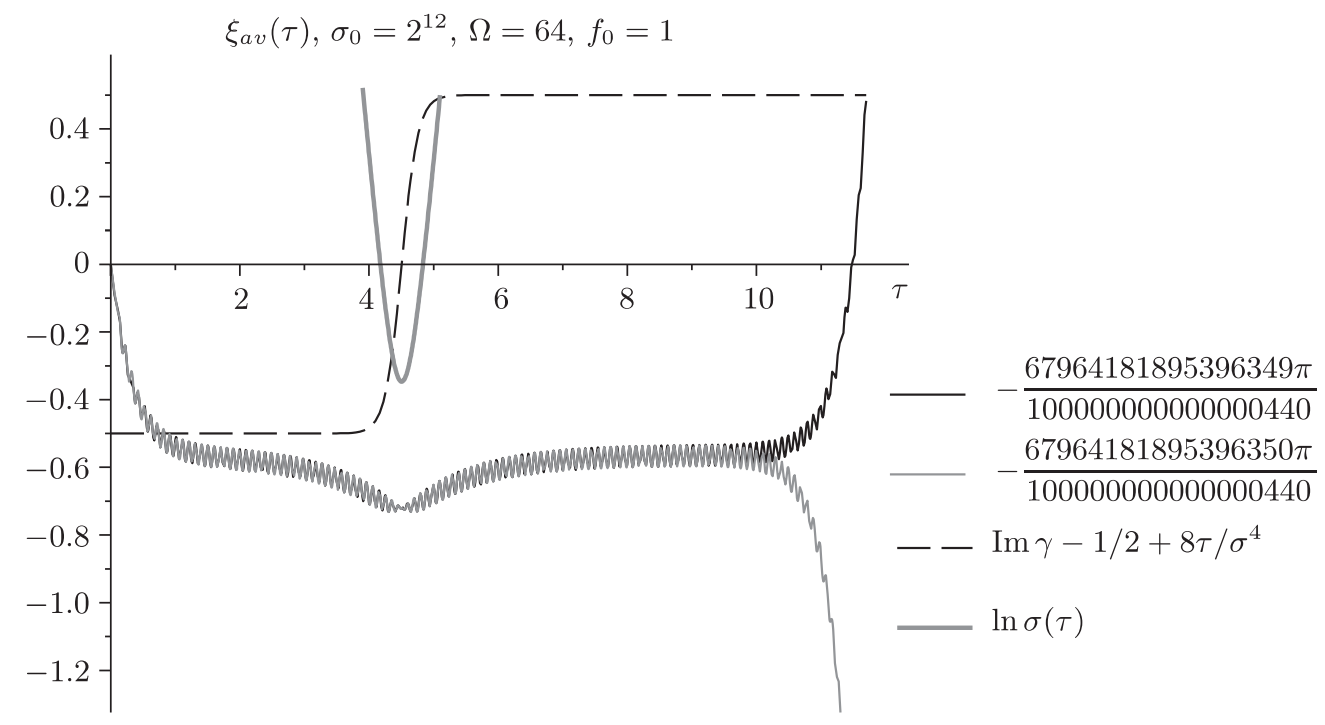

Рис. 4. Динамическая стабилизация центра пакета с $\sigma_{0}=2^{12}$ на высокой частоте $\Omega=64$.

положения на некоторую величину, тем меньшую, чем выше вынуждающая частота $\Omega$.

На высоких частотах $\Omega \sim 2^{7}$ и выше время стабилизации составляет порядка сотен/тысяч периодов вынуждающего воздействия, что может оказаться вполне достаточным для реконфигурации неустойчивых квантовых комплексов по другим координатам или спину под действием все того же периодического воздействия.

Асимптотическое поведение фаз $\varphi\left(\Omega, n=\log _{2} \sigma_{0} \rightarrow \infty\right)$, являющихся одновременно точками и стабилизации, и бифуркации, вполне соответствовало классическому характеру $\varphi_{\mathrm{cl}}(\Omega)=-\operatorname{arctg} \Omega$. Что же касается их $(\varphi)$ зависимостей от порядка $n$ начальной неопределенности координаты, то для ряда опробованных частот $\Omega$ они даже не были монотонными. Для важного случая "резонанса" $\Omega=1$ кривая $\varphi(n)$ хорошо удовлетворяла перевернутой зависимости Ферми-Дирака (см. рис. 7):

$$
\varphi=-\frac{\pi}{4}-\frac{\pi}{4\left(1+a 2^{n}\right)}, \quad a=0.076962(0.001744), \quad \operatorname{adj}-R^{2}=0.99965 .
$$

Вероятно, из-за ограниченной точности компьютерного продукта (Maple 17) или в силу иных причин не удалось зафиксировать интервал стабилизации центра, заметно превосходящий два основных "периода" $4 \pi / \omega$ даже при аномально больших начальных неопределенностях координаты, $\sigma_{0}=2^{25}$. Поэтому нельзя уверенно говорить о реализуемости неустойчивого предельного цикла в квантовой области, как это имеет место в классике. Но как уже отмечалось ранее, быстрое расплывание волны делает вопрос неактуальным.

Стоит обратить внимание на то, что в безразмерных переменных классическому пределу системы соответствует стремление безразмерной полуширины пакета к бесконечности, а не к нулю, о чем также свидетельствует поведение стабилизирующей фазы $\varphi\left(\Omega=1, n=\log _{2} \sigma_{0} \rightarrow \infty\right)$ (20) (см. рис. 7). Т. е. точному классическому 


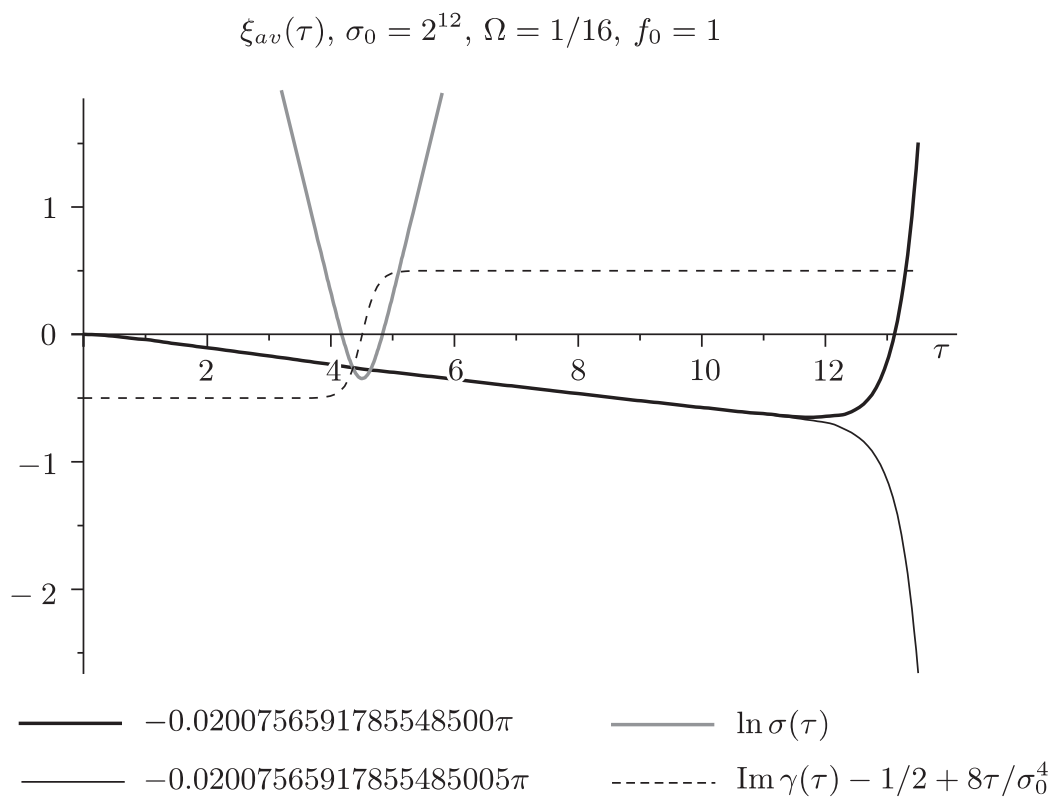

Рис. 5. Стабилизация на низкой частоте $\Omega=1 / 16$.

пределу отвечает бесконечный разброс координаты, и это никак не соответствует неустойчивому покою "шара на вершине горы" либо его вечному “закатыванию" на эту вершину. Фактически полученный результат свидетельствует о том, что квантовый перевернутый осциллятор не имеет классического аналога в строгом смысле этого слова. И тем он отличается от обычного линейного осциллятора, когерентные состояния которого соответствуют классическим гармоническим колебаниям. Можно только утверждать, что изученные состояния осциллятора перевернутого, сохраняя свой гауссовский тип и тем самым - фазовый объем, вполне законно претендуют на аналогичный статус [10].

\section{6. ЗАКЛЮЧЕНИЕ}

Исследована актуальная физическая система - перевернутый линейный осциллятор с гармоническим однородным возмущением. Установлено, что в ней с учетом квантовой специфики реализуется аналог неустойчивого предельного цикла, имеющего место для классического прототипа при начальных условиях, согласованных с частотой и начальной фазой синусоидального постоянного возмущения.

Показано, что в качестве стартовой в. ф. правомерен выбор комплекснозначной зависимости гауссовского типа с аномально большой неопределенностью координаты. Такие волновые пакеты, соединяя квантовым образом в единую трубку классические траектории с одинаковыми $p_{0}+q_{0}$, одновременно и удовлетворяют асимптотически оператору начальных условий, и обеспечивают аналитическую интегрируемость нестационарного УШ, притом в функциях того же типа. 


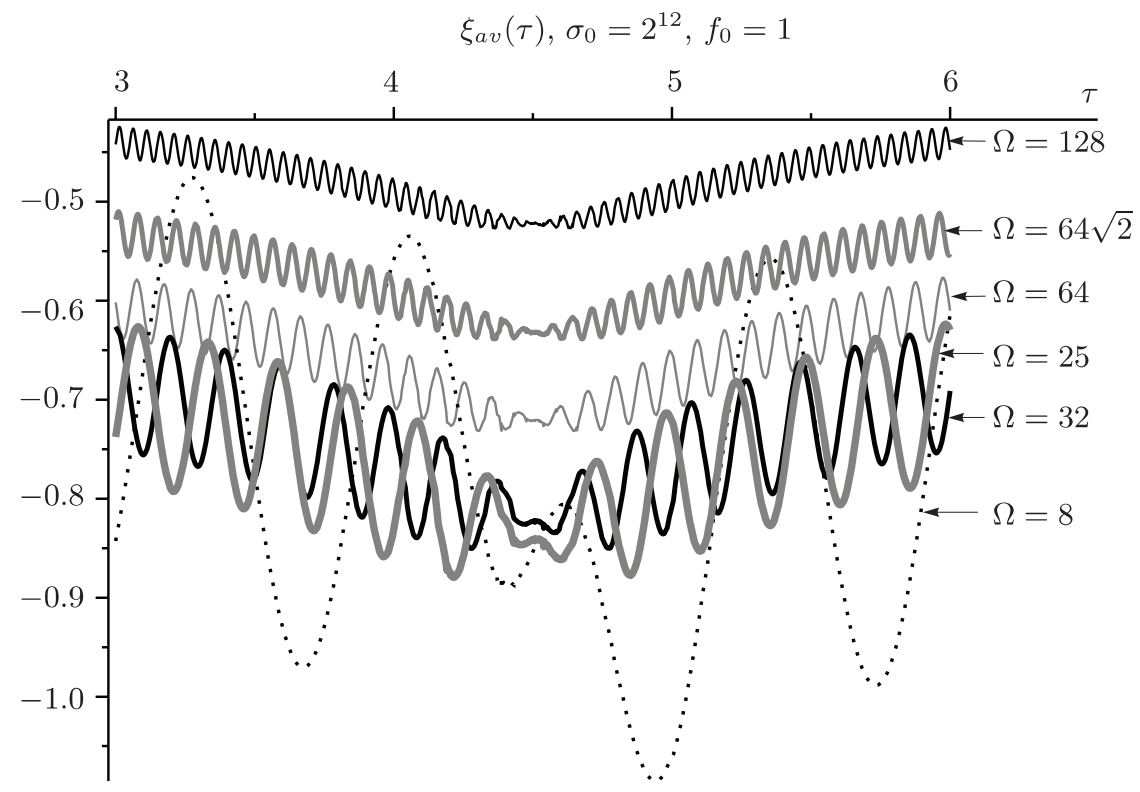

Рис. 6. Кривые $\overline{\xi(\tau)}$ в окрестности точки инверсии $\tau_{1}$ для различных вынуждающих частот $\Omega$.

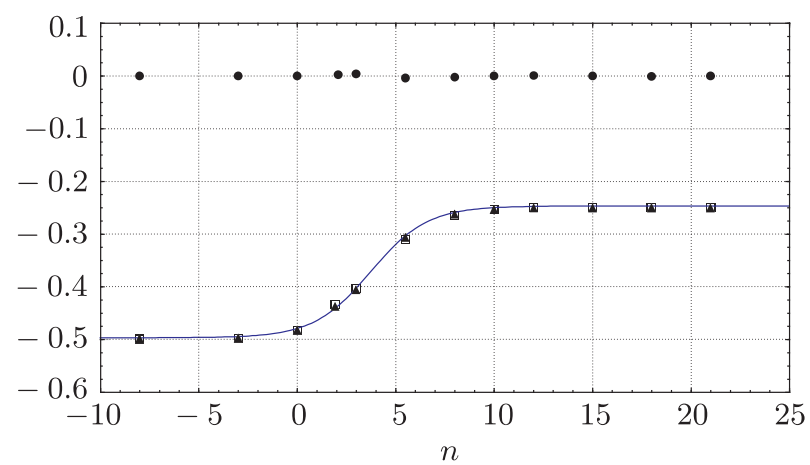

Рис. 7. Зависимость фазы стабилизации $\varphi$ и $n=\log _{2}\left(\sigma_{0}\right)$ (в ед. $\pi$ ) при $\Omega=1$ ( $\square$ - численное моделирование, $\boldsymbol{\Delta}$ - нелинейная регрессия (20), • погрешности, всё в единицах $\pi)$.

Для таких волновых пакетов обнаружено необычное явление компактизации в конфигурационном пространстве, причем для различающихся по величине квазимакроскопических начальных полуширин эффект имеет характер коллапса в. ф. в аномально узкий интервал неизменной длины порядка характерной единицы. И лишь потом пакет неограниченно расплывается.

Такое поведение неопределенности координаты у квантовой частицы позволяет визуализировать обнаруживаемый эффект временной стабилизации примерно на два естественных "периода" центра масс частицы волнового пакета вблизи положе- 
ния неустойчивого равновесия при определенных фазах периодического возмущения.

Как и в классической механике, эти стабилизирующие фазы служат одновременно точками бифуркации направления ухода волны направо/налево. Также зависимость фаз от вынуждаемой частоты тем ближе к классической формуле, чем больше начальная ширина волнового пакета.

Полученные результаты можно использовать при моделировании неустойчивых квантовомеханических систем в периодическом внешнем поле, например электромагнитном (лазерного излучения).

Исследованная проблема ставит комплекс разнообразных теоретических, экспериментальных и фундаментально научных вопросов, связанных как с реализацией исходной волновой функции, так и с эволюцией негауссовских пакетов и при обобщенном стабилизирующем воздействии (6), а также с осуществлением в случае перевернутого квантового осциллятора основополагающего принципа соответствия [6].

Благодарности. Автор выражает свою признательность профессору Норвежского университета науки и технологии К. Олауссену за проявленный интерес к проблеме и оказанные консультации.

\section{Список литературы}

[1] G. Barton, "Quantum mechanics of the inverted oscillator potential", Ann. Phys., 166:2 (1986), 322-363.

[2] S. Baskoutas, A. Jannussistl, R. Mignanig, "Dissipative tunnelling of the inverted CaldirolaKanai oscillator", J. Phys. A: Math. Gen., 27:6 (1994), 2189-2196.

[3] Sh. Matsumoto, M. Yoshimura, "Dynamics of barrier penetration in thermal medium: exact result for inverted harmonic oscillator", Phys. Rev. A, 63:1 (2000), 012104, 15 pp.

[4] Б. Н. Захарьев, “Дискретная и непрерывная квантовая механика, точно решаемые модели (Уроки квантовой интуиции II)”, ЭЧАЯ, 23:5 (1992), 1387-1468.

[5] C. A. Muñoz, J. Rueda-Paz, K. B. Wolf, "Discrete repulsive oscillator wavefunctions", J. Phys. A: Math. Theor., 42:48 (2009), 485210, 14 pp.

[6] M. Maamache, J.R. Choi, "Quantum-classical correspondence for the inverted oscillator", Chinese Phys. C, 41:11 (2017), 113106, 7 pp.

[7] P. Duclosi, E. Soccorsi, P. Šťovíček, M. Vittot, "On the stability of periodically time-dependent quantum systems", Rev. Math. Phys., 20:6 (2008), 725-764.

[8] Y. Nogami, F. M. Toyama, "Nonlinear Schrödinger soliton in a time-dependent quadratic potential", Phys. Rev. E, 49:5 (1994), 4497-4501.

[9] G.-J. Guo, Z.-Z. Ren, G.-X. Ju, X.-Y. Guo, "Quantum tunneling effect of a driven inverted harmonic oscillator", J. Phys. A: Math. Theor., 44:30 (2011), 305301, 16 pp.

[10] V. G. Bagrov, D. M. Gitman, E. S. Macedo, A. S. Pereira, "Coherent states of inverse oscillators and related problems", J. Phys. A: Math. Theor., 46:32 (2013), 325305, 13 pp.

Поступила в редакцию 5.02.2018, после доработки 19.03.2018, принята к печати 23.04.2018 